\title{
miR-101 inhibits cell proliferation by targeting Rac1 in papillary thyroid carcinoma
}

\author{
XIAOJIE LIN*, HONGYU GUAN* , HAI LI, LIEHUA LIU, JUAN LIU, \\ GUOHONG WEI, ZHIMIN HUANG, ZHIHONG LIAO and YANBING LI \\ Department of Endocrinology and Diabetes Center, The First Affiliated Hospital of \\ Sun Yat-sen University, Guangzhou, Guangdong 510080, P.R. China
}

Received September 27, 2013; Accepted October 21, 2013

DOI: $10.3892 /$ br.2013.192

\begin{abstract}
Accumulating evidence suggests that some microRNAs (miRNAs) are involved in papillary thyroid carcinoma (PTC) progression. However, it remains necessary to elucidate the underlying molecular mechanisms involved. In the present study, we investigated the role of microRNA-101 (miR-101) in PTC via targeting of Ras-related C3 botulinum toxin substrate 1 (Rac1). The results showed that miR-101 was significantly downregulated in PTC tissues compared with adjacent normal tissues. Restoration of miR-101 expression significantly inhibited cell proliferation in the K1 PTC cell line. Moreover, algorithm-based and experimental strategies verified Rac1 as a direct target of miR-101 in the K1 cell line. Taken together, these findings suggest that miR-101 inhibited PTC growth via the downregulation of Rac1 expression, providing a better understanding of miRNA-modulated signaling networks for future cancer therapeutics.
\end{abstract}

\section{Introduction}

Papillary thyroid carcinoma (PTC) is the most common pathological type of thyroid malignant tumor, accounting for $>80 \%$ of all thyroid cancers and typically occurs in young women or children (1). PTC usually presents as palpable thyroid mass or nodule and may be associated with hoarseness, dysphagia, stridor or pain (2). Although the majority of the PTCs have a good prognosis by surgical resection combined with radioiodine and levothyroxine treatment, metastasis and recurrence occur for certain PTCs (3). Certain clinical and pathological characteristics have been associated with a poor

Correspondence to: Professor Yanbing Li, Department of Endocrinology and Diabetes Center, The First Affiliated Hospital of Sun Yat-sen University, 58 Zhongshan Road II, Guangzhou, Guangdong 510080, P.R. China

E-mail: easd04lyb@126.com

*Contributed equally

Key words: microRNA-101, proliferation, thyroid cancer, Ras-related $\mathrm{C} 3$ botulinum toxin substrate 1 prognosis, such as advanced age at diagnosis, larger primary tumor ( $\geq 3 \mathrm{~cm}$ ), extrathyroidal invasion, lymph node metastasis and advanced tumor-node-metastasis (TNM) stage $(4,5)$. Therefore, a clear understanding of the pathogenesis of PTC is intrinsically valuable in the identification of novel diagnostic, prognostic and therapeutic targets.§

MicroRNAs (miRNAs) are a class of non-protein-coding RNAs that regulate gene expression at the post-transcriptional and translational levels (6). Accumulating evidence suggests that miRNAs play essential roles in the regulation of tumor cell proliferation, differentiation, apoptosis and metastasis (7). It is well known that microRNA-101 (miR-101) is a tumor-suppressive miRNA that is downregulated in several cancer types. For example, miR-101 was significantly downregulated in gastric cancer in comparison with normal gastric mucosas (8). Enhanced miR-101 expression suppressed colony formation ability as well as tumor cell motility of colorectal cancer (9). miR-101 expression is also involved in other cancer types including breast, liver and prostate cancer (10). However, the expression and biological functional roles of miR-101 in PTC are largely unknown.

The family of Ras homologue (Rho) plays a vital role in multiple cell functions as a molecular switch (11). Ras-related C3 botulinum toxin substrate 1 (Rac1), a member of the Rho family, plays a vital role in multiple cell functions including tumor cell proliferation, apoptosis, angiogenesis and metastasis (12). These findings underline the involvement of Rac1 in the development and progression of cancer types.

The role of miR-101 in PTC via targeting of Rac1 was therefore investigated. The results demonstrated that miR-101 was clearly downregulated in PTC tissue compared with that in adjacent normal thyroid tissue and restoration of miR-101 expression was able to reduce the proliferation of K1 PTC cells. Furthermore, we found that miR-101 is involved in PTC progression by directly targeting Racl. To the best of our knowledge, this is the first study to support the hypothesis that miR-101 downregulation is involved in the development of PTC.

\section{Materials and methods}

Cell culture. The K1 PTC cell line was purchased from the European Collection of Animal Cell Cultures (ECACC, 


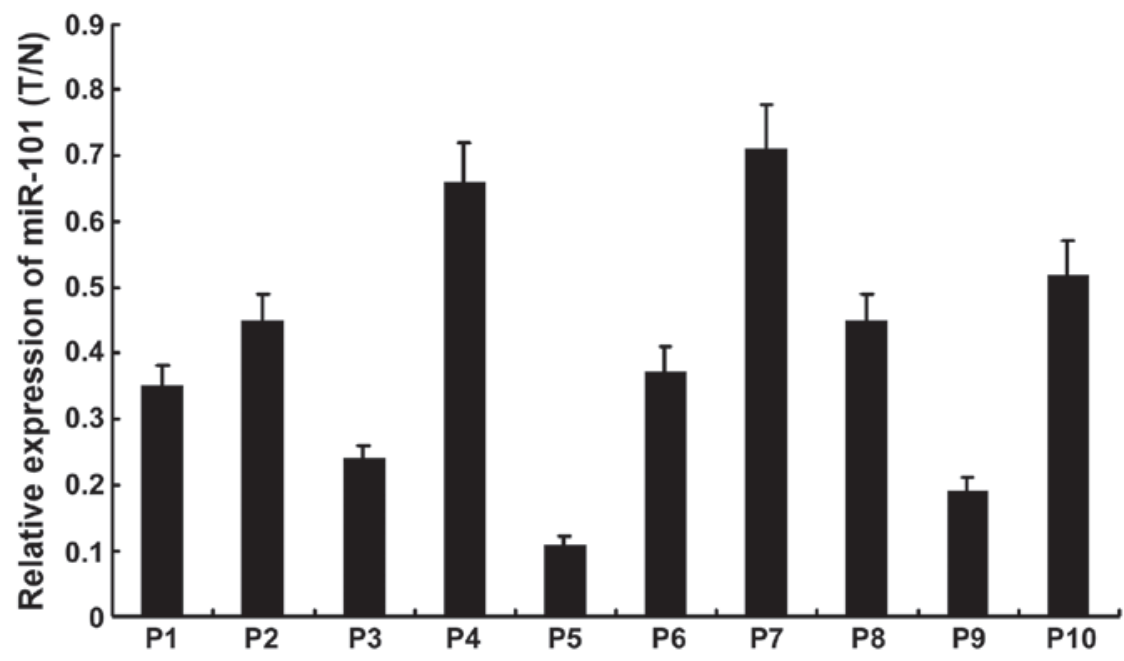

Figure 1. MicroRNA-101 (miR-101) is downregulated in papillary thyroid carcinoma (PTC). Mature miR-101 expression levels in PTC tissues and their paired non-malignant thyroid tissues were analyzed by quantitative reverse transcription-polymerase chain reaction analysis.

Salisbury, UK). The cell line was maintained in RPMI-1640 medium supplemented with $10 \%$ fetal bovine serum.

Patients and tissue specimens. A total of 10 cases of PTC, which had been clinically and histologically diagnosed at the First Affiliated Hospital of Sun Yat-sen University (China), were obtained following patient consent and approval from the Institutional Research Ethics Committee of Sun Yat-sen University.

Plasmids and constructs. pCMV-miR-101 was purchased from OriGene (Rockville, MD, USA). pCMV-hygro-Rac1 was purchased from Sino Biological (Beijing, China). 3'-UTRs of Rac1 were amplified and then cloned into a modified pGL3 control vector where SacII and PstI sites were introduced into the original $X b a \mathrm{I}$ site downstream of the luciferase gene. To mutate the binding sites within the 3'-UTR, a QuikChange site-directed mutagenesis kit from Stratagene (La Jolla, CA, USA) was used according to the manufacturer's instructions.

Western blot analysis. Cells were harvested, washed with $1 \mathrm{X}$ phosphate-buffered saline and lysed in mammalian protein extraction reagent RIPA (Beyotime, Jiangsu, China). A bicinchoninic acid protein assay kit (Pierce Biotechnology, Inc., Rockford, IL, USA) was used for protein quantification. Proteins were separated in SDS-PAGE, transferred to nitrocellulose membranes (Bio-Rad, Richmond, CA, USA) and immunoblotted with antibodies. The primary antibodies used were anti-Racl (Epitomics, Burlingame, CA, USA) and anti- $\alpha$-tubulin (Sigma-Aldrich, St. Louis, MO, USA).

RNA extraction and quantitative reverse transcription-polymerase chain reaction ( $R T-P C R)$. Total RNA was extracted from cells using TRIzol reagent (Invitrogen Life Technologies, Carlsbad, CA, USA), according to the manufacturer's instructions. The primers used for detection of mature miR-101 and U6 were designed by and purchased from Riobio Biotech Corporation (Guangzhou, China). The primers used for mRNAs were: Rac1, forward: 5'-CTGATGCAGGCCATCA AGT-3' and reverse: 5'-TCTCCAGGAAATGCATTGGT-3';
GAPDH, forward: 5'-TGGTGGACCTCATGGCCTAC-3' and reverse: 5'-CAGCAACTGAGGGCCTCTCT-3'.

Transient transfection. For siRNA transfection, Lipofectamine 2000 (Invitrogen Life Technologies) was used following the manufacturer's instructions. The siRNA sequence for Rac1 was 5'-GAGGAAGAGAAAAUGCCUG-3' (13).

MTTassay.Cellproliferationwasmeasuredby [3-(4,5-dimethylthiazol-2-yl)-2,5-diphenyltetrazolium bromide] (MTT) assay.

Colony formation. The indicated cells were plated in 6-well plates (500 cells per plate), cultured for 10 days and the colonies were stained with crystal violet.

Luciferase assay. Dual-luciferase reporter assays were performed as per the manufacturer's instructions (Promega Corporation, Madison, WI, USA), as previously described (14).

Statistical analysis. Data were expressed as mean \pm SD. Statistical significance was determined with t-test or analysis of variance by the SPSS 13.0 software. $\mathrm{P}<0.05$ was considered statistically significant difference.

\section{Results}

miR-101 is downregulated in PTC. To detect a possible involvement of miR-101 in PTC, a quantitative RT-PCR assay was performed to examine the expression level of miR-101 in 5 pairs of PTC tissues (T) and their adjacent non-cancerous thyroid tissues (N). As shown in Fig. 1, miR-101 was significantly downregulated in PTC tissues as compared with that in the adjacent non-cancerous thyroid tissues.

miR-101 inhibits $K 1$ cells proliferation. To investigate the possible function of miR-101 in PTC, we transfected miR-101 into the K1 PTC cell line and examined its effect on cell growth. Results of MTT assay showed that the restoration of miR-101 expression resulted in the inhibition of the growth of K1 cells 
A

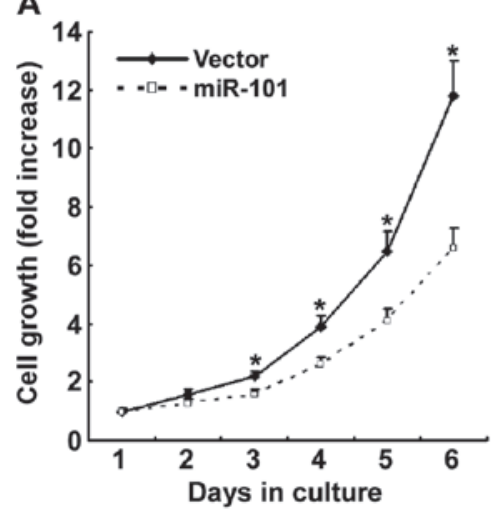

B

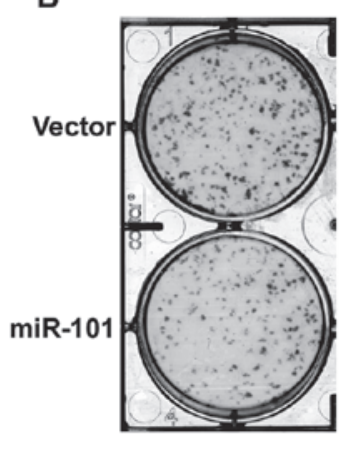

C

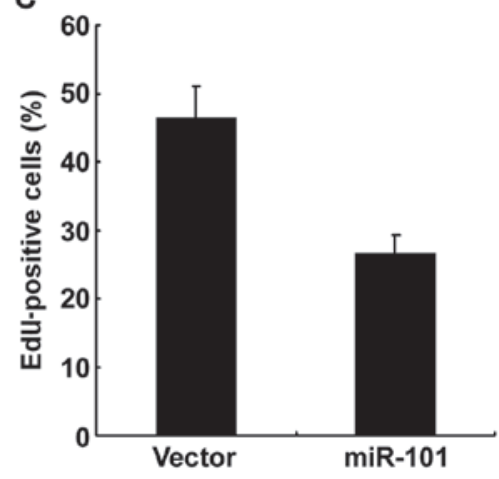

Figure 2. MicroRNA-101 (miR-101) inhibits K1 cell proliferation. (A) MTT assays were performed to investigate the effect of miR-101 on the proliferation of thyroid cancer cells at the indicated time points. ${ }^{*} \mathrm{P}<0.05$. (B) Colony formation assay of indicated cells. (C) Quantification of EdU incorporation assays.

\begin{abstract}
A
miR-101 AAGUCAAUAGUGUCAUGACAU 3'-UTR WT UCCCGACAUAACAUUGUACUGUA 3'-UTR Mut UCCCGACAUAACAUUGAUGACAA
\end{abstract}

B

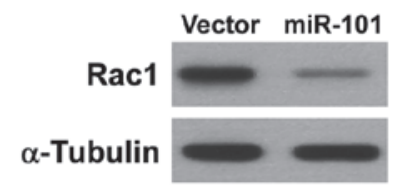

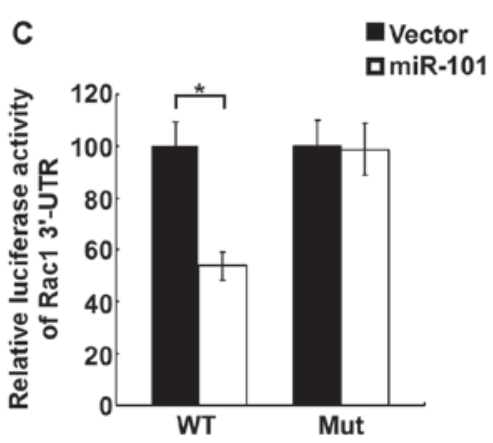

Figure 3. Rac1 is directly targeted by microRNA-101 (miR-101). (A) Schematic miR-101 putative target site in 3'-UTR of Rac1. (B) Western blot analysis of the protein levels of Rac1 in response to miR-101 overexpression. $\alpha$-tubulin was used as a loading control. (C) Relative luciferase activity in K1 cells transfected with wild-type (WT) or mutant (mut) Rac1 3'-UTR, as well as with miR-101 and a vector-control.

A

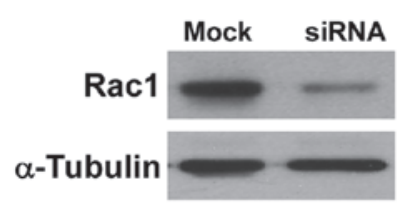

C

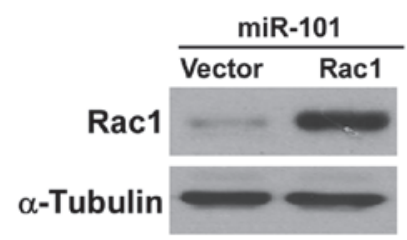

B

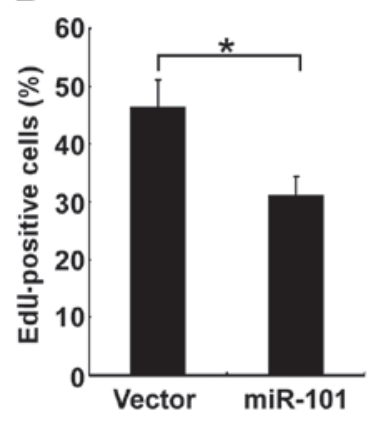

D

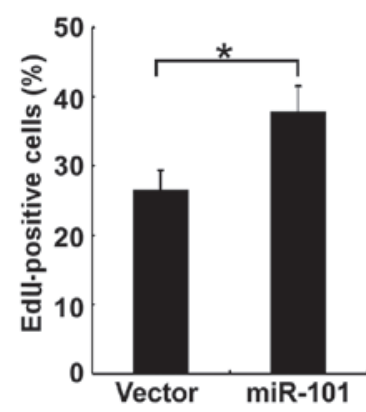

Figure 4. Rac1 is functionally related with the effect of microRNA-101 (miR-101). (A) Depletion of Rac1 and (B) inhibition of the proliferation of indicated cells. (C) Re-expression of Racl and (D) partially antagonized the miR-101-induced inhibition of cell proliferation.

as compared with the vector control (Fig. 2A). In addition, the colony formation assay exhibited that miR-101-overexpressing K1 cells generated a significantly lower number of colonies in comparison with vector control cells (Fig. 2B). The EdU incorporation assay revealed that the restored expression of miR-101 resulted in reduced proliferation of thyroid cancer cells (Fig. 2C). These data demonstrate miR-101 is involved in thyroid cancer cell proliferation.

miR-101 directly targets Racl expression in PTC. To explore the mechanism of miR-101-induced cell growth inhibition, we searched putative targets of miR-101 using TargetScan (http://www.targetscan.org/) and miRanda (www.microrna. org/miranda_new.html). The conserved target gene Rac1 was identified and used for subsequent investigation (Fig. 3A). The protein level of Rac1 was significantly downregulated in miR-101-overexpressing K1 cells (Fig. 3B), whereas no significant change in the mRNA levels of Racl was observed (data not shown). Luciferase reporter assays were performed to investigate the direct interaction between miR-101 and Rac1. As shown in Fig. 3C, ectopic expression of miR-101 significantly inhibited the luciferase acitivity of a reporter construct containing the 3'-UTR Rac1. Moreover, when a mutation was introduced into the predicted miR-101 site in 3'-UTR Rac1, the reporter failed to response to miR-101, suggesting the silencing was associated with the predicted target site. Taken together, these data suggest that Rac1 is a direct target of miR-101. 
Racl is functionally related with the effect of miR-101. In order to investigate the role of Racl in PTC cell proliferation, we knocked down the expression of Rac1 in K1 cells (Fig. 4A). It was shown that the suppression of miR-101 led to the inhibition of cell proliferation (Fig. 4B). Moreover, re-expression of Rac1 in miR-101-expressing K1 cells (Fig. 4C) partially antagonized the miR-101-induced inhibition of cell proliferation (Fig. 4D. These data suggest that Rac1 is a functionally relevant target of the effect of miR-101 on PTC cell proliferation.

\section{Discussion}

A vital biological feature of tumors is their potential for unrestrained growth. The activation of oncogenes and the aberrant expression of tumor suppressor genes are the main factors leading to tumors (15). miRNAs are small, single-stranded RNAs that regulate gene expression (16). In recent studies, miRNAs have been known to be involved in the pathogenesis of cancer and miRNA expression profiles are associated with prognosis in various types of cancer (17). miRNAs with increased expression levels in tumors may function as oncogenes and promote cancer development by negatively regulating tumor suppressor genes. By contrast, the miRNAs frequently downregulated in cancer may function as tumor suppressor and inhibit cancer development by downregulating oncogenes (18). miR-101 is a well-known tumor suppressive miRNA that is downregulated in several cancer types. Nevertheless, the expression and biological function of miR-101 in PTC remains to be clarified.

Liu et al (19) have identified that 248 miRNAs were significantly deregulated $(\mathrm{P}<0.01)$ in $\mathrm{PTC}$ tissues when compared with their matching normal thyroid tissues. Subsequently, hsa-miR-101 was identified. Consistent with results of that study, we found that miR-101 was downregulated in PTC, suggesting that miR-101 is a tumor suppressor. Furthermore, we investigated the role of miR-101 in the growth of PTC cells by MTT assay, colony formation and EdU incorporation assay. As expected, the ectopic expression of miR-101 inhibited the growth of PTC cells. Our results therefore indicate that miR-101 is involved in the negative regulation of PTC cell proliferation. However, the mechanism underlying the effect of miR-101 on tumor growth remains to be elucidated.

Target genes of miR-101 were identified to examine the molecular mechanism underlying the miR-101-induced suppression of PTC cell growth. According to the in silico analyses, we predicted Racl as a direct target, which encodes a small G-protein that is an important member of the Rho family (20). Rac1 is overexpressed in various types of cancer including breast, colon and lung carcinomas (21-23) and participates in all stages of tumor formation (24). However, the effect of Racl on PTC remains preliminary and whether miR-101 affects the growth of PTC cells through Rac1 remains to be determined. We have clearly demonstrated that miR-101 induced the inhibition of PTC cell proliferation by directly targeting Rac1. Depletion of Rac1 mimics the anti-proliferative effect of miR-101, indicating that Rac1 has a functional contribution to PTC cell proliferation. The results also showed that suppression of Rac1 cannot fully recapitulate the miR-101 effect on PTC cell proliferation and together with the fact that many other target genes modulated by miR-101 are found in other types of cancer, additional studies should be conducted to investigate other target genes regulated by miR-101 that contribute to PTC tumorigenesis.

Accumulated evidence have identified miRNAs that aid in the diagnosis of disease and serve as potential targets for therapy in the future (25). Recent successful preclinical therapeutic trials in cancers include miR-380-5p replacement in neuroblastoma (26) and $\mathrm{miR}$ replacement/anti-miR combination therapy in hepatoblastoma (27). Notably, certain miR-based therapies have been applied in the clinic, including blockade of miR-122 for chronic viral hepatitis (28-29). Our findings provide insight into the therapeutic implications of miR-101 in PTC.

\section{Acknowledgements}

This study was supported by grants from the 5010 project of Sun Yat-sen University (no. 2010002), the Science and Technology Fund of Guangzhou (no. 1346000270), the Industrial Technology Research and Development Funds of Guangdong Province, the Natural Science Foundation of China (no. 81370076) and the Guangdong Provincial Key Laboratory of Medicine.

\section{References}

1. Lloyd RV, Buehler D and Khanafshar E: Papillary thyroid carcinoma variants. Head Neck Pathol 5: 51-56, 2011.

2. Singh A, Butuc R and Lopez R: Metastatic papillary thyroid carcinoma with absence of tumor focus in thyroid gland. Am J Case Rep 14: 73-75, 2013.

3. Silver CE, Owen RP, Rodrigo JP, et al: Aggressive variants of papillary thyroid carcinoma. Head Neck 33: 1052-1059, 2011.

4. Voutilainen PE, Multanen MM, Leppaniemi AK, et al: Prognosis after lymph node recurrence in papillary thyroid carcinoma depends on age. Thyroid 11: 953-957, 2001.

5. Chou CK, Chen RF, Chou FF, et al: miR-146b is highly expressed in adult papillary thyroid carcinomas with high risk features including extrathyroidal invasion and the BRAF(V600E) mutation. Thyroid 20: 489-494, 2010.

6. Chen PS, Su JL and Hung MC: Dysregulation of microRNAs in cancer. J Biomed Sci 19: 90, 2012.

7. O'Day E and Lal A: MicroRNAs and their target gene networks in breast cancer. Breast Cancer Res 12: 201, 2010.

8. Carvalho J, van Grieken NC, Pereira PM, et al: Lack of microRNA-101 causes E-cadherin functional deregulation through EZH2 up-regulation in intestinal gastric cancer. J Pathol 228: 31-44, 2012.

9. Chandramouli A, Onyeagucha BC, Mercado-Pimentel ME, et al: MicroRNA-101 (miR-101) post-transcriptionally regulates the expression of EP4 receptor in colon cancers. Cancer Biol Ther 13: 175-183, 2012.

10. Wang R, Wang HB, Hao CJ, et al: MiR-101 is involved in human breast carcinogenesis by targeting Stathmin1. PLoS One 7: e46173, 2012.

11. Fritz G, Just I and Kaina B: Rho GTPases are over-expressed in human tumors. Int J Cancer 81: 682-687, 1999.

12. Silva AL, Carmo F and Bugalho MJ: RAClb overexpression in papillary thyroid carcinoma: a role to unravel. Eur J Endocrinol 168: 795-804, 2013.

13. Lewis-Saravalli S, Campbell S and Claing A: ARF1 controls Rac1 signaling to regulate migration of MDA-MB-231 invasive breast cancer cells. Cell Signal 25: 1813-1819, 2013.

14. Guan H, Wei G, Wu J, et al: Down-regulation of miR-218-2 and its host gene SLIT3 cooperate to promote invasion and progression of thyroid cancer. J Clin Endocrinol Metab 98: E1334-El1344, 2013.

15. Liu D, Xia P, Diao D, et al: MiRNA-429 suppresses the growth of gastric cancer cells in vitro. J Biomed Res 26: 389-393, 2012.

16. Bartel DP: MicroRNAs: target recognition and regulatory functions. Cell 136: 215-233, 2009. 
17. Lee YS and Dutta A: MicroRNAs in cancer. Annu Rev Pathol 4: 199-227, 2009

18. Zhang B, Pan X, Cobb GP and Anderson TA: microRNAs as oncogenes and tumor suppressors. Dev Biol 302: 1-12, 2007.

19. Liu X, He M, Hou Y, et al: Expression profiles of microRNAs and their target genes in papillary thyroid carcinoma. Oncol Rep 29: 1415-1420, 2013.

20. Wu YJ, Tang Y, Li ZF, Li Z, Zhao Y, Wu ZJ and Su Q: Expression and significance of Rac1, Pak1 and Rock1 in gastric carcinoma. Asia Pac J Clin Oncol: Jan 8, 2013 (Epub ahead of print). doi: 10.1111/ajco.12052.

21. Schnelzer A, Prechtel D, Knaus U, et al: Rac1 in human breast cancer: overexpression, mutation analysis, and characterization of a new isoform, Raclb. Oncogene 19: 3013-3020, 2000.

22. Jordan P, Brazao R, Boavida MG, Gespach C and Chastre E: Cloning of a novel human Raclb splice variant with increased expression in colorectal tumors. Oncogene 18: 6835-6839, 1999.

23. Liu Y, Wang Y, Zhang Y, et al: Abnormal expression of p120-catenin, E-cadherin, and small GTPases is significantly associated with malignant phenotype of human lung cancer. Lung Cancer 63: 375-382, 2008.
24. Bosco EE, Mulloy JC and Zheng Y: Rac1 GTPase: a 'Rac' of all trades. Cell Mol Life Sci 66: 370-374, 2009.

25. Sempere LF: Integrating contextual miRNA and protein signatures for diagnostic and treatment decisions in cancer. Expert Rev Mol Diagn 11: 813-827, 2011.

26. Swarbrick A, Woods SL, Shaw A, et al: miR-380-5p represses p53 to control cellular survival and is associated with poor outcome in MYCN-amplified neuroblastoma. Nat Med 16: 1134-1140, 2010

27. Cairo S, Wang Y, de Reynies A, et al: Stem cell-like micro-RNA signature driven by Myc in aggressive liver cancer. Proc Nat Acad Sci USA 107: 20471-20476, 2010.

28. Cho WC: MicroRNAs in cancer - from research to therapy. Biochim Biophys Acta 1805: 209-217, 2010.

29. Wahid F, Shehzad A, Khan T and Kim YY: MicroRNAs: synthesis, mechanism, function, and recent clinical trials. Biochim Biophys Acta 1803: 1231-1243, 2010. 